\title{
Dip Slide Culture of Intraoperative Peritoneal Irrigation Fluid for Prediction of Septic Complication in Elective Colorectal Surgery
}

\author{
BERNDT E. B. CLAESSON ${ }^{1 *}$ AND DAN E. W. HOLMLUND ${ }^{2}$ \\ Department of Clinical Bacteriology ${ }^{1}$ and Department of Surgery, ${ }^{2}$ Kärnsjukhuset, 54185 Skövde, Sweden
}

Received 19 May 1986/Accepted 26 August 1986

\begin{abstract}
A controlled prospective study of a simplified technique, the dip slide culture method, for assessment of bacterial concentration in peritoneal irrigation fluid at the end of an elective colorectal operation is presented. The prediction of postoperative surgical infection based on intraoperative culture was compared between this method and a standard streak-plate technique in 190 patients. One gram of metronidazole was given intravenously as prophylaxis on induction of anesthesia and $12 \mathrm{~h}$ postoperatively. Intraoperative growth of members of the family Enterobacteriaceae or Staphylococcus aureus was strongly correlated to infection $(P<$ 0.001). By using this finding as a single criterion for the prediction of sepsis, sensitivity and specificity for the dip slide method were $\mathbf{7 0 . 8}$ and $94.3 \%$, respectively, compared with 79.2 and $94.6 \%$, respectively, for the streak-plate method. Of the 24 infections, $20(83.3 \%)$ were correctly predicted when a combination of the two methods was used. An increasing number of Enterobacteriaceae or $S$. aureus in the dip slide culture resulted in a steady rise in the rate of infections from $5.7 \%$ at 0 CFU to $57 \%$ at $\geq 80$ CFU compared with $4.1 \%$ at 0 $\mathrm{CFU} / \mathrm{ml}$ and $45.4 \%$ at $\geq 800 \mathrm{CFU} / \mathrm{ml}$ with the streak-plate method. The differences were statistically significant at the $\geq 5$ CFU level with regard to wound infection $(P<0.001)$ and deep surgical infection $(P<0.01)$. It is concluded that the dip slide is a simple, rapid, and reliable method for the routine assessment of bacterial contamination in colorectal operations.
\end{abstract}

The development of sepsis after bowel surgery is undoubtedly a dynamic process in which a number of factors interact. A rough division into management factors (i.e., timing of surgery and surgical technique) and factors that are patient related (age, diagnosis, etc.) can be made. However, the type and number of contaminating bacteria at the time of operation are the indisputable critical factors with which the current study is concerned.

Two previous investigations of bowel operations demonstrated that the likelihood of infection was greatly increased when certain densities of Escherichia coli and Staphylococcus aureus were present in peritoneal fluid before closure, as judged by the streak-plate technique (5) and dip slide culture (4). Because the concept of a critical level of contamination was based on a small number of patients $(4,5)$, the purpose of this study was to reevaluate the criteria given above by comparing the predictive value of the dip slide method with that of the standard streak-plate technique in a large controlled study of elective colorectal surgery in which metronidazole was given to all patients for protection against anaerobic sepsis.

\section{MATERIALS AND METHODS}

Patients. In this study were included all patients undergoing elective colorectal surgery at the surgical clinics at Kärnsjukhuset, Skövde, Sweden, and Bassjukhuset, Lidköping, Sweden, during a 30-month period, with the following exceptions: antimicrobic medication within a week before surgery, closure of colostomy, and preexisting infection. A total of 243 patients were enrolled in the study. Five patients were excluded due to incorrect protocol (three patients) and infection recognized at the time of operation (two patients).

\footnotetext{
* Corresponding author
}

Intraoperative cultures were related to potential risk factors in 238 patients. However, analysis of the correlation between the degree of contamination and the development of infection was restricted to 190 patients, since 12 were given antimicrobic medication for urinary tract infection or pneumonia within 5 days after the operation and 36 patients were randomized to receive cefuroxime postoperatively; the results in the latter group are presented in a separate report in conjunction with details of preparation and surgical routines (B. E. B. Claesson, S. Filipsson, D. E. W. Holmlund, T. W. Mätzsch, and L. Wählby, Br. J. Surg., in press).

Antimicrobic prophylactic regime. All patients were given $1 \mathrm{~g}$ of metronidazole intravenously $30 \mathrm{~min}$ preoperatively and $12 \mathrm{~h}$ after the operation.

Intraoperative bacteriological sampling. Before the wound was closed, the abdominal cavity was washed three times with saline $(0.85 \% \mathrm{NaCl}) ; 500 \mathrm{ml}$ was used for each washing. After the last irrigation, the top lid of a dip slide culture plate (Uricult; Orion Diagnostica, Trosa, Sweden) was detached with forceps and the plate was dipped into the remaining fluid at the bottom of the peritoneal cavity. The culture plate was then put in its original container and transported to the laboratory for incubation.

Aspiration of $5 \mathrm{ml}$ of irrigation fluid from the same site was then carried out with a syringe. This sample was emptied into a rubber membrane-sealed glass bottle filled with $\mathrm{CO}_{2}$.

Samples from Kärnsjukhuset reached the laboratory within $10 \mathrm{~min}$ and were immediately processed. Specimens from Bassjukhuset, however, arrived $18 \mathrm{~h}$ postoperatively; in the meantime the dip slide was incubated at $37^{\circ} \mathrm{C}$ and the sample of irrigation fluid was kept at $4^{\circ} \mathrm{C}$. Controls of this delayed processing showed that colony count of irrigation fluid with Escherichia coli, Staphylococcus aureus, Streptococcus faecalis, and Bacteroides fragilis showed a variance of less than $\pm 1 \log _{10}$ unit when kept at $4^{\circ} \mathrm{C}$ for $24 \mathrm{~h}$. 
Sampling at septic sites. Discharges from wounds were sampled with cotton swabs and transported in Stuart medium to the laboratory. Specimens from deep-sited foci were collected with a syringe and transported in sealed $\mathrm{CO}_{2-}$ gassed bottles. At least three blood cultures were taken when septicemia was suspected. Aerobic and anaerobic biphasic blood culture bottles were used (Institute of Medical Microbiology, University of Göteborg, Göteborg, Sweden).

Culture methods. The dip slide was originally designed for the screening of urinary tract infection (9). It consists of a rectangular plastic central plate with two agar surfaces; Cled and MacConkey. Cled (cysteine-lactose-electrolytedeficient) agar is designated for the cultivation of aerobic and facultatively anaerobic pathogens (12). The lack of electrolytes inhibits the swarming of Proteus spp. Lactosefermenting organisms are recognized by alterations in color from green to yellow. MacConkey agar allows the growth of gram-negative rods but inhibits gram-positive cocci. The area of each agar side on the dip slide used here is $10 \mathrm{~cm}^{2}$. The amount of fluid (urine) taken up by one agar side is 0.01 to $0.02 \mathrm{ml} \mathrm{(6)}$.

The dip slide cultures were incubated aerobically at $37^{\circ} \mathrm{C}$ for $18 \mathrm{~h}$. The number of $\mathrm{CFU}$ on the Cled agar side was recorded. Colony count was restricted to members of the family Enterobacteriaceae and Staphylococcus aureus (see definitions below).

For comparison of various potential risk factors, patients were divided into three degrees of contamination on the basis of dip slide colony count of Enterobacteriaceae and Staphylococcus aureus, i.e., 0, 1 to 4 , or $\geq 5$ CFU.

Tenfold serial dilutions of the $\mathrm{CO}_{2}$-bottle contents $(0.1 \mathrm{ml})$ were streaked onto culture plates. Columbia agar (lab m; Ford Lane, Salford, England) supplemented with $7.5 \%$ human blood and $1 \mathrm{mg}$ of menadione (E. Merck AG, Darmstadt, Federal Republic of Germany) per $100 \mathrm{ml}$ was used for both aerobic and anaerobic (Gas Pak; BBL Microbiology Systems, Cockeysville, Md.) incubation. An additional selective anaerobic plate contained neomycin $(100$ $\mu \mathrm{g} / \mathrm{ml}$; Sigma Chemical Co., St. Louis, Mo.). Anaerobic plates were prereduced. Aerobic plates were incubated for a minimum of 2 days and anaerobic plates for a minimum of 5 days. Two sets of plates were used for all dilution steps, and colony count was expressed as the mean of two culture plates in the dilution yielding 30 to $300 \mathrm{CFU}$ (11).

For cultures of material obtained from septic lesions, aerobic and anaerobic cultures were done on the nonselective media described above. Thioglycolate broth (BBL) was also used. Anaerobic plates were supplemented with $30-\mu \mathrm{g}$ gentamicin disks (AB Biodisk, Solna, Sweden) after plating. These plates were incubated in an anaerobic cabinet (Don Whitley, Scientific Ltd., Baildon, Shipley, West Yorkshire, England).

Blood culture bottles were inspected twice daily for 7 days before discarding. Subculturing of primary isolates was done on Columbia agar with $7.5 \%$ human blood and on GC agar supplemented with hemoglobin $(1 \mathrm{~g} / 100 \mathrm{ml})$ and IsoVitaleX enrichment $(1 \mathrm{ml} / 100 \mathrm{ml} ; \mathrm{BBL})$. Plates were incubated aerobically with and without $5 \% \mathrm{CO}_{2}$ and in an anaerobic cabinet.

The intraoperative culture results were known only by a research technician at the Department of Clinical Bacteriology, Kärnsjukhuset, and were kept secret until after the evaluation of septic complications.

Identification of bacterial isolates. After the 18-h incubation period, dip slide colonies grown on the Cled agar side were
Gram stained and tested for catalase and, if necessary, oxidase and coagulase activity (7). A preliminary allocation to Enterobacteriaceae (oxidase-negative, gram-negative rods) or Staphylococcus aureus (catalase- and coagulasepositive, gram-positive cocci) was then made. Bacteria not meeting these criteria were disregarded.

Identification of isolates obtained from the intraoperative aspiration procedure and from blood cultures and wound discharges was accomplished by standard procedures adopted from Cowan and Steel (7), as was definite typing of isolates obtained by the dip slide procedure. In addition, aerobic and facultatively anaerobic gram-negative rods and streptococci were identified to the species level with the Analytical Profile Index System for Enterobacteriaceae and streptococci (Analytab Products, Plainview, N.Y.). Biochemical identification of anaerobes was done with the Minitek system (BBL), and gas-liquid chromatography of glucose end products was done according to the Virginia Polytechnic Institute manual (10).

Antimicrobic susceptibility testing. Anaerobic bacteria isolated from postoperative infections were tested for susceptibility to metronidazole (AB Leo, Helsingborg, Sweden). The agar dilution method was employed (8), using PDM agar (AB Biodisk) with $7 \%$ human blood. The inoculum was $10^{6}$ $\mathrm{CFU} / \mathrm{ml}$. Overnight incubation was carried out in an anaerobic cabinet at $37^{\circ} \mathrm{C}$. Plates were read only when a control plate clearly showed visible growth. The breakpoint was $8 \mu \mathrm{g} / \mathrm{ml}$.

Registration of complications. Patients were observed over a 4-week period for complications from infection, which were noted by a surgeon in collaboration with a ward nurse. Wound infection was defined as described by Pollock (14). Deep surgical infection denoted intraabdominal sepsis or septicemia (spiking fevers and growth of intestinal bacteria in blood culture in the absence of urinary tract infection or pneumonia).

Statistical methods. The Pitman nonparametric test (3) was used to test the correlation between the degree of contamination and the potential risk factors listed in Table 1 . This test was also used for evaluating the impact of degree of contamination on the development of sepsis. The comparison in sepsis rate between patients whose irrigation fluids grew Enterobacteriaceae or Staphylococcus aureus and those that did not was done with the Fisher exact test. Only two-sided tests were used.

\section{RESULTS}

Streak-plate culture of irrigation fluid. The recovery rate of strictly anaerobic strains in relation to the total number of strains was similar in the two hospitals, $36.8 \%$ at Kärnsjukhuset and $41.7 \%$ at Bassjukhuset, despite the 24-h time lag before plating in the latter case. It therefore was decided to present the bacteriological findings from the two surgical clinics together.

A total of 454 strains representing 74 different species were cultured in 169 of $238(71 \%)$ patients. A majority of strains $(283 ; 62 \%)$ were strictly anaerobic species, but the single most frequently isolated species was Escherichia coli $(n=90)$, followed by Bacteroides vulgatus $(n=53)$, Bacteroides distasonis $(n=38)$, Bacteroides fragilis $(n=$ $20)$, Streptococcus faecalis $(n=16)$, Staphylococcus epidermidis $(n=16)$, Bacteroides oralis $(n=15)$, Bacteroides ruminicola $(n=14)$, Eubacterium lentum $(n=11)$, and Peptostreptococcus anaerobius $(n=10)$.

The major groups of bacteria and their respective concen- 
TABLE 1. Description and risk factor analysis of 238 patients by degree of contamination as judged by dip slide colony count of Enterobacteriaceae or Staphylococcus aureus

\begin{tabular}{|c|c|c|c|c|c|}
\hline \multirow{2}{*}{ Parameter } & \multicolumn{4}{|c|}{ No. of CFUs } & \multirow{2}{*}{$P$ value if $<0.05^{b}$} \\
\hline & $0(n=133)$ & $1-4(n=34)$ & $\geq 5(n=35)$ & $\geq 5(n=36)^{a}$ & \\
\hline \multicolumn{6}{|l|}{ Age (yr) } \\
\hline Mean & 60.4 & 63.1 & 61.7 & 64.4 & \\
\hline Range & $15-86$ & $33-84$ & $24-85$ & $20-86$ & \\
\hline Sex (no. male/no. female) & $71 / 62$ & $17 / 17$ & $20 / 16$ & $22 / 13$ & \\
\hline \multicolumn{6}{|l|}{ No. with the following diagnoses: } \\
\hline Malignancy & 85 & 27 & 22 & 24 & \\
\hline Inflammatory bowel disease & 25 & 5 & 5 & 4 & \\
\hline Others & 23 & 2 & 8 & 8 & \\
\hline \multicolumn{6}{|l|}{ Weight index $\left(\mathrm{kg} / \mathrm{m}^{2}\right)^{c}$} \\
\hline Mean & 23.8 & 24.0 & 24.3 & 25.0 & \\
\hline SD & 4.2 & 3.4 & 3.3 & 5.4 & \\
\hline \multicolumn{6}{|l|}{ Preoperative hospital stay (days) } \\
\hline Mean & 5.8 & 9.3 & 5.1 & 5.4 & \\
\hline Range & $1-25$ & $2-66$ & $1-15$ & $1-20$ & \\
\hline \multicolumn{6}{|l|}{ No. with the following surgical procedures: } \\
\hline Right hemicolectomy & 55 & 12 & 10 & 8 & \\
\hline Left hemicolectomy & 30 & 10 & 8 & 9 & \\
\hline Anterior resection with stomy & 9 & 4 & 5 & 5 & \\
\hline Anterior resection without stomy & 6 & 2 & 0 & 4 & \\
\hline Abdominoperineal resection & 18 & 2 & 5 & 3 & \\
\hline Proctocolectomy & 3 & 1 & 3 & 1 & \\
\hline Others & 12 & 3 & 4 & 6 & \\
\hline \multicolumn{6}{|l|}{ Operating time $(\mathrm{h})$} \\
\hline$<3$ & 102 & 30 & 33 & 29 & \\
\hline $3-4$ & 24 & 4 & 1 & 6 & \\
\hline$>4$ & 7 & 0 & 1 & 1 & \\
\hline \multicolumn{6}{|l|}{ No. with: } \\
\hline Fecal contamination & 14 & 3 & 7 & 6 & \\
\hline Abdominal drains & 5 & 2 & 5 & 5 & 0.014 \\
\hline Decompressive colostomy & 12 & 4 & 10 & 10 & $<0.001$ \\
\hline Antimicrobial therapy within 5 days of surgery & 10 & 1 & 1 & 0 & \\
\hline
\end{tabular}

${ }^{a}$ Group randomized to receive cefuroxime (see text).

${ }^{b}$ All cultures with $\geq 5 \mathrm{CFU}$ were pooled.

c Body weight (kilograms) divided by height squared.

trations in irrigation fluid are presented in Fig. 1. Occasionally, anaerobic gram-negative rods were present in numbers exceeding $10^{5} \mathrm{CFU} / \mathrm{ml}$, but the vast majority of the strains were found in a range from 10 to $999 \mathrm{CFU} / \mathrm{ml}$.

Dip slide culture of irrigation fluid. The separation of patients into various categories of contamination, based on the dip slide colony count of Enterobacteriaceae and Staphylococcus aureus, was as follows. Negative dip slide culture results were seen in 133 patients, whereas 1 to 4 CFU was recorded in 34 patients, and $\geq 5 \mathrm{CFU}$ was recorded in 71 patients.

Staphylococcus aureus was recovered from only four patients and in low numbers, (range, 2 to $27 \mathrm{CFU}$ ), while Enterobacteriaceae $(n=109)$ was seen in 101 patients (range, 1 to $343 \mathrm{CFU}$ ). Escherichia coli was the most abundant member of the family $(n=94)$, followed by Klebsiella pneumoniae $(n=10)$.

Comparison between dip slide and streak-plate quantitation. Dip slide colony count corresponded to a wide range in CFU per milliliter, as determined by the streak-plate method. Dip slide cultures with $\geq 5$ CFU of Staphylococcus aureus or Enterobacteriaceae were mainly seen at intervals of $\geq 100 \mathrm{CFU} / \mathrm{ml}$ (Fig. 2). When the analysis was restricted to patients with positive streak-plate $(x)$ as well as positive dip slide $(y)$ cultures, the correlation coefficient between the two variables was 0.67 . The corresponding linear regression function was $\log y=0.53 \log x-0.22$, with a standard deviation of 1.13 .

Septic complications and the relation to intraoperative cultures. A total of 24 infections were seen in 190 patients; 8 infections (11.3\%) at Bassjukhuset, 16 infections (10.3\%) at Kärnsjukhuset.

A species-related approach was first investigated. A comparison in sepsis rate was made of patients with irrigation fluids that did and those that did not grow Enterobacteriaceae or Staphylococcus aureus. By the dip slide technique $25.4 \%$ (17 of 67 ) of patients with a positive intraoperative culture of either of these microbes were infected and 5.7\% (7 of 123) of those with negative culture or growth of species other than Enterobacteriaceae or Staphylococcus aureus were infected, a difference of $19.7 \pm 11.2 \%$ (95\% confidence interval; $P<0.001)$. The corresponding figures for the streak-plate method were $27.9 \%(19$ of 68$)$ and $4.1 \%$ (5 of $122)$, a difference of $23.8 \pm 11.2 \%(P<0.001)$. The predic- 


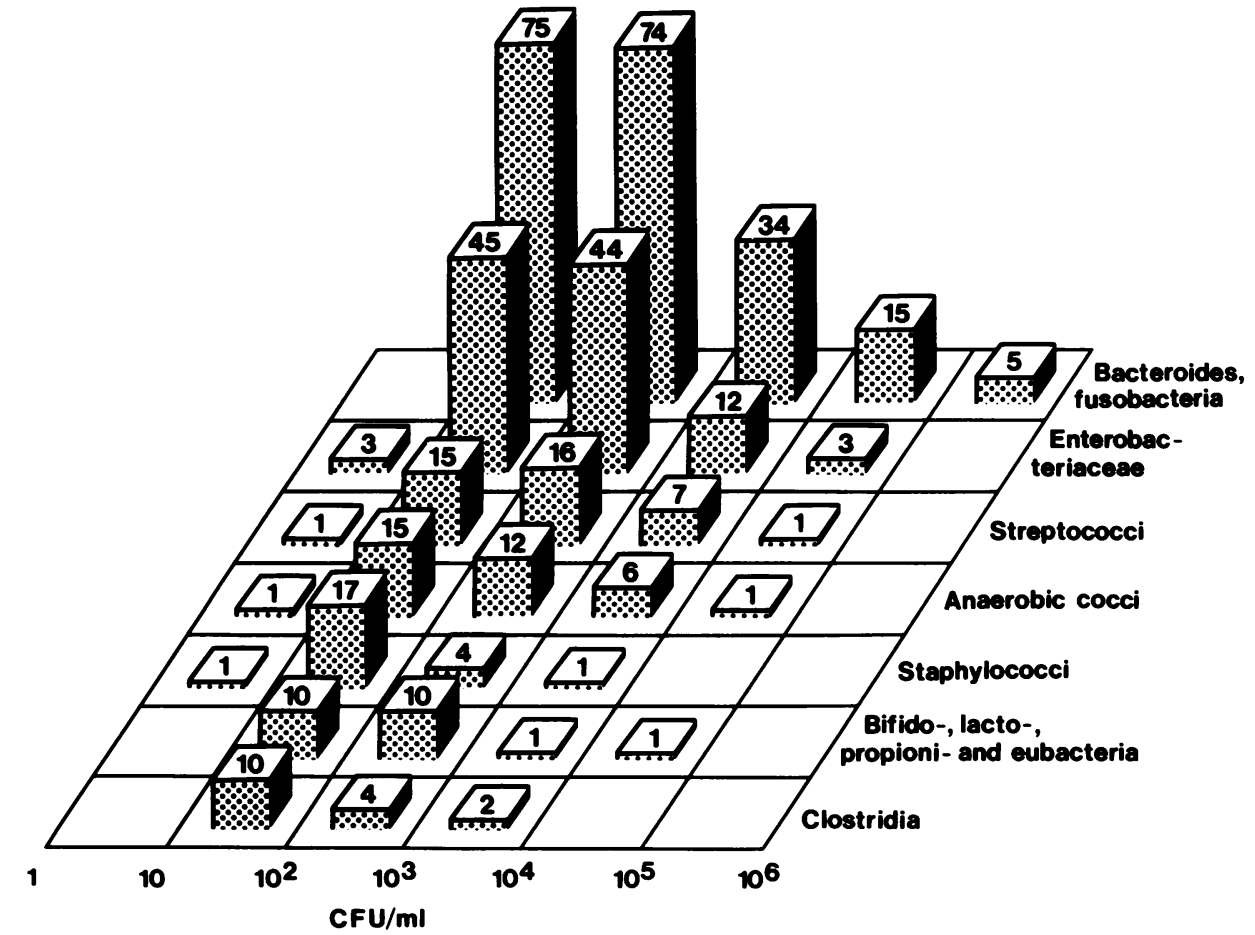

FIG. 1. Major groups of bacteria and their respective concentrations in peritoneal irrigation fluid. The value at the top of each box denotes the number of strains isolated at a particular concentration.

tion of sepsis, based solely on the presence of these microbes in peritoneal irrigation fluid, yielded a $70.8 \%$ sensitivity for the dip slide technique compared with $79.2 \%$ for the standard streak-plate technique. The respective specificities were 94.3 and $94.6 \%$. By combining the two methods, 20 of the 24 infections $(83.3 \%)$ were predicted.

Second, a com!ined quantitative and species-related approach showed th $t$ the increasing number of postoperative infections seen in contamination categories with higher dip slide colony connt: of Enterobacteriaceae or Staphylococcus aureus reached statistical significance regarding both local wound sepsis $(P<0.001)$ and deep surgical sepsis $(P<$ 0.01 ) at the $\geq 5 \mathrm{CFU}$ level (Table 2). The probability of infection also increased stepwise above this level (Fig. 3A). The highest probability score $(0.57)$ was seen at colony counts above $80 \mathrm{CFU}$.

A similar graph was obtained by the streak-plate method. The maximum risk level $(0.45)$ was seen at $\geq 800$ CFU of Enterobacteriaceae or Staphylococcus aureus per ml (Fig. 3B).

By means of streak-plate total colony count of aerobic and anaerobic bacteria, patients were allocated to various levels

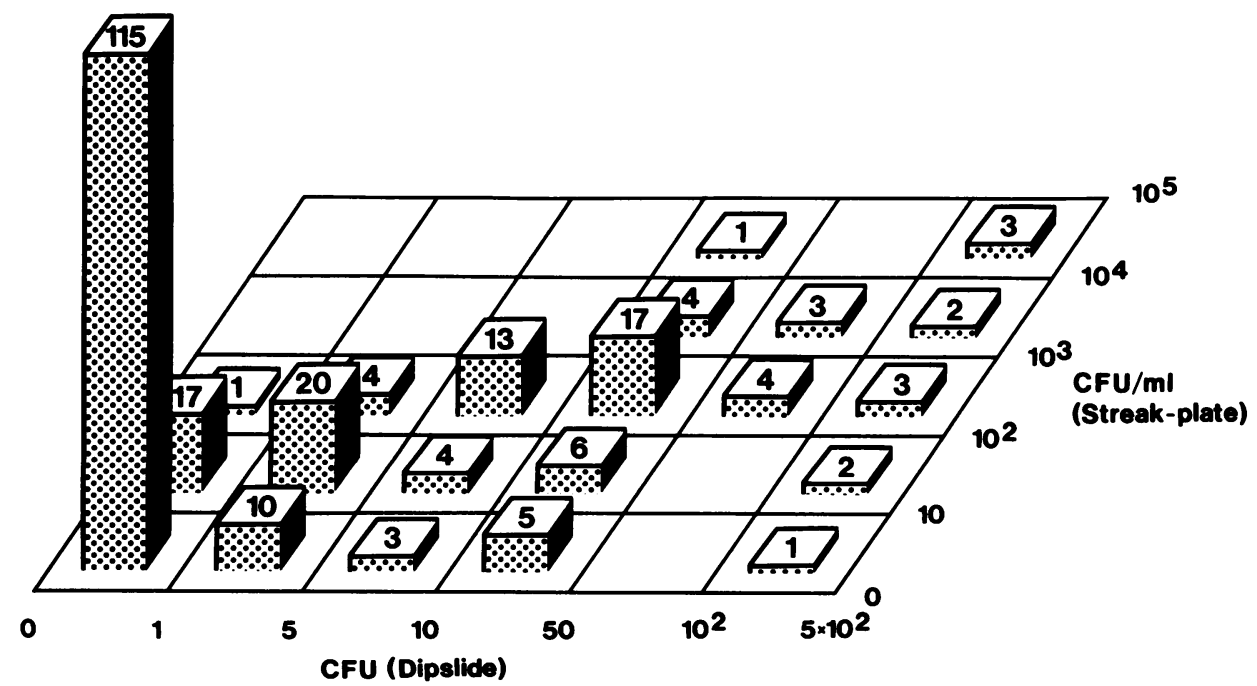

FIG. 2. Comparison of quantitation of Enterobacteriaceae and Staphylococcus aureus by the dip slide and streak-plate methods in 238 patients. 
TABLE 2. Postoperative infections in relation to intraoperative dip slide colony count of Enterobacteriaceae or Staphylococcus aureus in 190 patients

\begin{tabular}{lcccc}
\hline & \multicolumn{3}{c}{$\begin{array}{c}\text { No. of patients (incidence of infection [\%] } \\
\text { with: }\end{array}$} & \\
\cline { 2 - 4 } Infection & $\begin{array}{c}0 \text { CFU } \\
(n=123)\end{array}$ & $\begin{array}{c}1-4 \text { CFU } \\
(n=33)\end{array}$ & $\begin{array}{l}\geq 5 \mathrm{CFU} \\
(n=34)\end{array}$ & $P$ value \\
\hline $\begin{array}{c}\text { Local wound } \\
\text { sepsis }\end{array}$ & $4(3.3)$ & $3(9.1)$ & $8(23.5)$ & $<0.001$ \\
$\begin{array}{c}\text { Deep surgical } \\
\text { sepsis }\end{array}$ & $3(2.4)$ & $2(6.1)$ & $6(17.6)$ & $<0.01$ \\
$\begin{array}{c}\text { All surgical } \\
\text { sepsis }\end{array}$ & $7(5.7)$ & $3^{a}(9.1)$ & $14(41.2)$ & $<0.001$ \\
\hline
\end{tabular}

${ }^{a}$ Two patients with deep surgical sepsis also had local wound sepsis.

of intraoperative contamination (Fig. 4). This strictly quantitative analysis showed that there was no significant difference in the rate of infections when patients with low colony counts were compared with those with high colony counts.

Responsible pathogens in septic complications. From one patient with a local wound infection, Bacteroides vulgatus and Fusobacterium varium were isolated together with Escherichia coli. All other septic abdominal wounds solely grew aerobes or facultative anaerobes. Bacteroides fragilis and Escherichia coli were recovered from two blood cultures in a patient with septicemia. Intraabdominal infection never grew anaerobes, while a mixed aerobic and anaerobic flora was cultured from two fistulas and two perineal wounds. Anaerobic strains isolated from septic sites remained fully susceptible to metronidazole (MIC, $\leq 8 \mu \mathrm{g} / \mathrm{ml}$ ).

The dip slide showed growth of bacteria in 16 of 21 cases in which cultures from infections were available. In 13 of 16
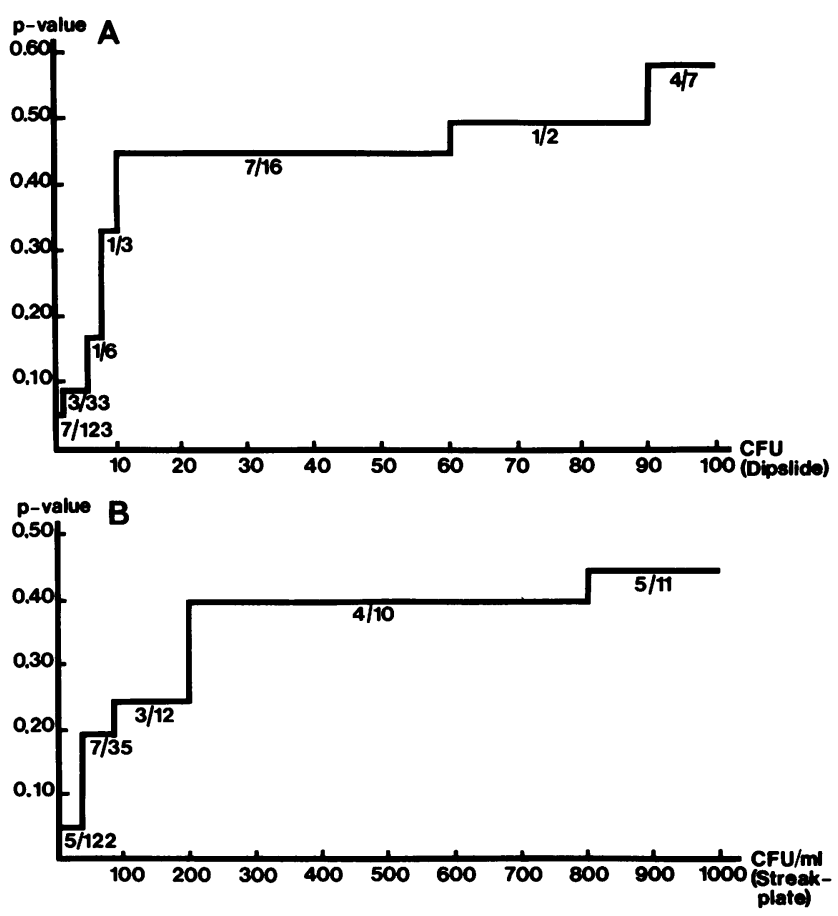

FIG. 3. The probability of infection was estimated as a function of the number of CFU (by the dip slide method [A]) and CFU/ml (by the streak-plate method [B]) of Enterobacteriaceae or Staphylococcus aureus under nondecreasing restriction.

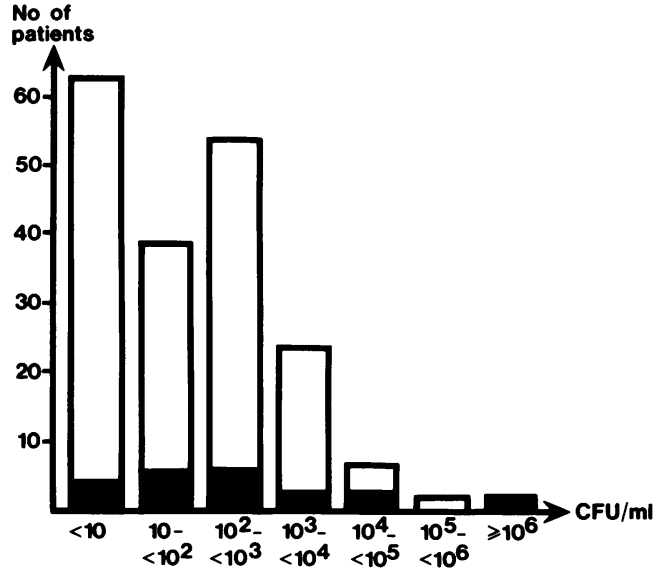

FIG. 4. The distribution of 190 patients at various degrees of contamination as judged by the total number of bacteria in intraoperative irrigation fluid (streak-plate method) and the relation to postoperative infection. Symbols: $\square$, no infection; $\mathbf{\square}$, infection.

cases at least one species of the type found during the operation was also isolated from the septic focus.

With the aspiration technique, the intraoperative culture had at least one species in common with the postoperative culture in 15 of 18 cases. However, only 17 of 36 isolates from postoperative septic lesions had previously been captured from the intraoperative samples. Four species were represented among these 17 strains: Escherichia coli, Klebsiella pneumoniae, Staphylococcus aureus, and Streptococcus pyogenes.

Bacteria found at septic sites but not at the respective operation were Enterobacter cloacae, Proteus vulgaris, Providentia rettgeri, Staphylococcus epidermidis, Streptococcus faecalis, Streptococcus milleri/S. intermedius, Bacteroides fragilis, other Bacteroides, spp., and Fusobacterium varium.

The culture results from the four patients with infections not predicted by either of the methods were Klebsiella pneumoniae and Staphylococcus epidermidis (wound infection), Escherichia coli (wound infection), and Staphylococcus epidermidis (repeatedly isolated from a subphrenic abscess in a patient splenectomized en passant); and in the fourth case cultures were not taken since pus from an intraabdominal abscess emptied via a colostomy.

Clinical details in relation to dip slide culture. The degree of contamination was not significantly correlated to age, sex, diagnosis, body weight index, preoperative hospital stay, surgical procedure, or operating time (Table 1). Only 13 of 30 patients with fecal contamination, as judged by the operating surgeon, had a dip slide score of $\geq 5 \mathrm{CFU}$. The correlation between visible and bacteriological contamination was thus indeterminate. Abdominal drains, however, were overrepresented in patients with $\geq 5 \mathrm{CFU}(P=0.014)$, as were decompressive colostomies $(P<0.001)$.

\section{DISCUSSION}

The use of wound irrigation for predicting postoperative infections in colorectal surgery has been reported both to fail (2) and to succeed (15). A stepwise quantitative correlation was found among colonic, peritoneal, and wound bacterial counts, using a 10-ml volume for irrigation (15); if $\geq 100$ $\mathrm{CFU} / 10 \mathrm{ml}$ was present in the peritoneal or in the wound 
washout, the infection rate was 57 and $64 \%$, respectively, compared with 3 and $6 \%$ if $<100 \mathrm{CFU} / 10 \mathrm{ml}$ was present. An increasing rate of infections with higher numbers has also been reported with a $100-\mathrm{ml}$ peritoneal washout (18).

In a previous study, which mainly included young patients who were operated on for inflammatory bowel disease, we investigated the peritoneal density of bacteria in the first and third washouts of three irrigations with saline, $500 \mathrm{ml}$ each time (5). If at least $50 \mathrm{CFU}$ of either Escherichia coli or Staphylococcus aureus per $\mathrm{ml}$ was found, $90 \%$ of the patients became infected compared with $9 \%$ if these species were present at lower numbers.

A combined qualitative (species related) and quantitative assessment of wound contamination was further evaluated in a study of elective colorectal procedures, in which no antibiotic prophylaxis was used in controls (4). Before closure, a dip slide was dipped into the remaining peritoneal fluid without a preceding irrigation. If patients within the control group had at least 5 CFU of either Enterobacteriaceae or Staphylococcus aureus on their dip slide, $100 \%$ became infected compared with $27 \%$ at lower counts; in three of four patients with local wound sepsis in the lowcount category, pus solely grew Bacteroides fragilis.

In the present study, a striking predominance of anaerobes was seen in the intraoperative cultures. Nevertheless, only a single case of abdominal wound infection involving strictly anaerobic bacteria was recorded. The efficacy of a shortterm perioperative course of treatment with metronidazole for prevention of anaerobic sepsis is in line with previous experience (19). Anaerobic bacteria recovered postoperatively remained susceptible to this agent. Apparently, however, metronidazole did not prevent the development of infections in patients whose irrigation cultures grew Enterobacteriaceae or Staphylococcus aureus. These results are in conflict with the concept that an effective agent directed against either the aerobic or the anaerobic microflora is enough to prevent infection by eliminating bacterial synergism.

Disregarding strains that were present only at the operation or only at the septic site in patients with infections, and instead ranking those which were present both intraoperatively and postoperatively as significant contributors to sepsis, only 4 species of 74 encountered in the study met the criteria: Escherichia coli, Klebsiella pneumoniae, Staphylococcus aureus, and Streptococcus pyogenes.

In addition to these species-related aspects, the study clearly demonstrates an increase in the likelihood of infection with increasing numbers of Enterobacteriaceae or Staphylococcus aureus in the peritoneal fluid. This was shown both by the dip slide and the streak-plate methods. The correlation between contamination level and infection was not limited to local wound sepsis $(P<0.001)$, but was also true for deep surgical sepsis $(P<0.01)$. This is interesting since intraabdominal infections traditionally are thought to emanate from anastomotic breakdown on a surgicotechnical basis.

A strictly quantitative assessment of the total bacterial contamination failed to identify patients who were at risk for infection. This may reflect the killing of potentially harmful anaerobes with metronidazole.

Open drains and a colostomy constituted two of four factors which predisposed to infection in a recent large uniand multivariate analysis of nonselected colorectal operations (16). In this context, it is noteworthy that the performance of a decompressive colostomy and abdominal drains were the only potential risk factors that were over- represented in patients with a high level of bacteriologic contamination in this study. It may therefore be suggested, although it is not proven, that either of these measures are prone to create a dense bacterial contamination in the operative field, in turn jeopardizing and, in some cases, overwhelming the host defenses.

The prediction of infection based on the intraoperative recovery of Enterobacteriaceae and Staphylococcus aureus did not differ significantly between the two methods. This is somewhat surprising, since one would expect the sensitivity of the detection level to increase when larger volumes were used for culture: the streak-plate procedure actually involves the assessment of 5 to 10 times more fluid than the dip slide culture. It was difficult, however, to establish the exact amount of fluid cultured by the dip slide method, since the relation between the two methods was not described by a straight line. Moreover, the resulting regression function showed a wide confidence interval. In this study we did not elucidate whether this distribution was due to variation in irrigation fluid viscosity leading to a variation in the volume cultured on the dip slide, crowding of colonies or competition of nutrients at higher densities, or inborn statistical error associated with low dip slide colony counts.

The accuracy of the dip slide method for establishing a diagnosis of urinary tract infection has been questioned (17), and the method is referred to as semiquantitative (1). Other investigators report an accurate viable count in ranges of $>10^{4} \mathrm{CFU} / \mathrm{ml}(6)$, and in a large study it was considered as accurate as the pour-plate technique and more accurate and reliable than the conventional standard loop technique (13).

At present the dip slide method is widely used throughout the world for screening purposes, often by personnel with no special bacteriologic skills. This is possible since the pathogens usually causing the infection are carefully described on a chart supplied by the manufacturer in conjunction with a photograph of typical colonies both on Cled and MacConkey agar. For routine surveillance of intraoperative contamination, this information should be combined with Gram staining and testing for oxidase, coagulase, and catalase activity. Members of the family Enterobacteriaceae and Staphylococcus aureus are thereby rapidly discriminated from irrelevant bacteria.

An objective method obviously is needed because the opinion of the surgeon of fecal soilage was only weakly associated with the culture results. The identification of high-risk patients by the dip slide method and the selective administration of a short-term cefuroxime course of therapy to such patients resulted in a significant reduction in the expected rate of infections (Claesson et al., in press).

In conclusion, intraoperative bacterial contamination is an important factor contributing to sepsis. By the irrigation technique, $83.3 \%$ of the postoperative infections were predictable. A combined species-related and quantitative approach was more accurate than either of the other two methods for prediction of infection. The dip slide method is characterized by speed, simplicity, and good economy for assessment of intraoperative contaminants that contribute significantly to postoperative infection. The reliability is comparable to that of the standard streak-plate technique.

\section{ACKNOWLEDGMENTS}

We acknowledge the laboratory assistance of Charlotta Littmarck, medical technician at the Department of Clinical Bacteriology, Kärnsjukhuset, Skövde, and Anders Odén, P1 8414, Kungälv, who planned and performed the statistical analysis. 


\section{LITERATURE CITED}

1. Barry, A. L., P. B. Smith, and M. Turck. 1975. Cumitech 2, Laboratory diagnosis of urinary tract infections. Coordinating ed., T. L. Gavan. American Society for Microbiology, Washington, D.C.

2. Bartlett, J. G., R. E. Condon, S. L. Gorbach, J. S. Clarke, R. L. Nichols, and O. Shigeru. 1978. Veterans Administration Cooperative Study on bowel preparation for elective colorectal operations: impact of oral antibiotic regimen on colonic flora, wound irrigation cultures and bacteriology of septic complications. Ann. Surg. 188:249-254.

3. Bradley, J. W. 1968. Distribution-free statistical tests. PrenticeHall, London.

4. Claesson, B., Å. Brandberg, and H. Brevinge. 1981. Selected postoperative antibiotic prophylaxis in colo-rectal surgery on the basis of bacterial contamination in the operative field. Acta Chir. Scand. 147:289-293.

5. Claesson, B., Å. Brandberg, L. O. Nilsson, and N. G. Kock. 1981. Quantitative recovery of contaminating bacteria at operation and the relation to postoperative infection in intestinal surgery. Acta Chir. Scand. 147:285-288.

6. Cohen, S. N., and E. H. Kass. 1967. A simple method for quantitative urine culture. N. Engl. J. Med. 277:176-180.

7. Cowan, S. T., and K. J. Steel. 1974. Manual for the identification of medical bacteria, 2nd ed. Cambridge University Press, London.

8. Ericsson, H. M., and J. Sherris. 1971. Report of an international collaborative study. Acta Pathol. Microbiol. Scand. Sect. B. 217(Suppl.):11-12.

9. Guttman, D., and G. R. E. Naylor. 1967. Dip-slide: an aid to quantitative urine culture in general practice. $\mathrm{Br}$. Med. $\mathrm{J}$. 3:343-345.

10. Holdeman, L. V., E. C. Cato, and W. E. C. Moore (ed.). 1977.
Anaerobe laboratory manual, 4th ed. Virginia Polytechnic Institute and State University, Blacksburg.

11. Koch, A. L. 1981. Growth measurement, p. 185-187. In P. Gerhardt, R. G. E. Murray, R. N. Costilow, E. W. Nester, W. A. Wood, N. R. Krieg, and G. B. Phillips (ed.), Manual of methods for general bacteriology. American Society for Microbiology, Washington, D.C.

12. Mackey, J. P., and G. H. Sandys. 1965. Laboratory diagnosis of infections of the urinary tract in general practice by means of a dip-inoculum transport medium. Br. Med. J. 2:1286-1288.

13. McAllister, T. A., G. C. Arneil, W. Barr, and P. Kay. 1973. Assessment of plane dipslide quantitation of bacteriuria. Nephron 11:111-122.

14. Pollock, A. V. 1979. Surgical wound sepsis. Lancet ii:1283-1286.

15. Scheibel, J. H., M. Lykkegaard Nielsen, and T. Wamberg. 1978. Septic complications in colorectal surgery after 24 hours versus 60 hours of preoperative antibiotic bowel preparation. Acta Chir. Scand. 144:527-532.

16. Simchen, E., M. Shapiro, T. G. Sacks, J. Michel, A. Durst, and Z. Eyal. 1984. Determinants of wound infection after colon surgery. Ann. Surg. 199:260-265.

17. Taylor, W. I. 1971. Rapid detection of bacteria in urine. Lab. Med. 2:29.

18. Törnqvist, A., G. Ekelund, A. Forsgren, L. Leandoer, S. Olson, and J. Ursing. 1981. Single dose doxycykline prophylaxis and peroperative bacteriological culture in elective colorectal surgery. Br. J. Surg. 68:565-568.

19. Willis, A. T., I. R. Ferguson, P. H. Jones, K. D. Phillips, P. V. Tearle, R. V. Fiddian, D. F. Graham, D. H. C. Harland, D. F. R. Hughes, D. Knight, W. M. Mee, N. Pashby, R. L. RothwellJackson, A. K. Sachdeva, L. Sutch, C. Kilbey, and D. Edwards. 1977. Metronidazole in prevention and treatment of bacteroides infection in elective colonic surgery. Br. Med. J. 1:607-610. 